\title{
Right-Handed Sneutrino as Cold Dark Matter of the Universe
}

\author{
Takehiko Asaka, ${ }^{1}$ Koji Ishiwata, ${ }^{2}$ and Takeo Moroi $^{2}$ \\ ${ }^{1}$ Institut de Théorie des Phénomènes Physiques, EPFL, CH-1015 Lausanne, Switzerland \\ ${ }^{2}$ Department of Physics, Tohoku University, Sendai 980-8578, Japan
}

(Dated: December, 2006)

\begin{abstract}
We consider the minimal supersymmetric standard model (MSSM) extended by introducing three right-handed (s)neutrinos to account for neutrino masses in the oscillation experiments. Assuming that the neutrino masses are purely Dirac-type, the lightest right-handed sneutrino $\tilde{\nu}_{R}$ can be the lightest superparticle (LSP), which is a good candidate of cold dark matter (CDM) of the universe. We study the possibility of realizing $\tilde{\nu}_{R}$-CDM, paying a special attention to the production of $\tilde{\nu}_{R}$ via decay of the next-to-lightest superparticle (NLSP) after its freeze-out time. It is shown that the late decay of the MSSM-LSP (the LSP among superparticles in the MSSM) can produce a sufficient amount of $\tilde{\nu}_{R}$ to explain the observed dark-matter density, and that the $\tilde{\nu}_{R}$-CDM scenario can be realized in a wide range of parameter space. We also consider the constraint on the decay of MSSM-LSP from the big-bang nucleosynthesis (BBN); we found that the case with stau being the MSSM-LSP is severely constrained.
\end{abstract}

PACS numbers: 14.60.Pq, 12.60.Jv, 95.35.+d, 98.80.Cq

\section{INTRODUCTION}

Supersymmetry is one of the most attractive candidates of physics beyond the standard model since it may solve various open questions in particle physics. It can solve the hierarchy and naturalness problems and also it can realize gauge-coupling unification. Supersymmetric theories have a great advantage in cosmology as well. This is because it can provide a viable candidate of cold dark matter (CDM) of the universe which cannot be understood in the framework of the standard model of particle physics. The lightest supersymmetric particle (LSP) becomes stable with $R$-parity conservation and can be CDM. Recently, the relic density of CDM in the present universe has been precisely determined by the WMAP observation [1]:

$$
\Omega_{\mathrm{DM}} h^{2}=0.105_{-0.013}^{+0.007},
$$

where $h \simeq 0.73[1]$ is the present Hubble constant in units of $100 \mathrm{~km} / \mathrm{sec} / \mathrm{Mpc}$. Any candidate of CDM must explain this dark matter density. Crucial questions left to us are, then, what is the LSP for CDM and how they are produced accounting for (11) in the history of the universe. So far, various scenarios have been discussed in literatures [2].

In Ref. [3], we have proposed a scenario that the lightest right-handed sneutrino $\tilde{\nu}_{R}$ is the LSP and is CDM of the universe. ${ }^{1}$ This is motivated by very small but non-vanishing neutrino masses strongly suggested by the experimental evidences of neutrino oscillations (see, e.g., Refs. [6, 7, 8, 9, 10]). The non-zero neutrino masses require physics beyond the standard model. The simplest way to generate neutrino masses is probably to introduce

1 The possibility of the right-handed sneutrino as CDM has also been discussed recently in different context. See Refs. [4, [5]. right-handed neutrinos (as well as sneutrinos in supersymmetric theories).

It has been widely discussed that right-handed (s)neutrinos are introduced together with their superheavy Majorana masses which are much larger than the electroweak scale $\sim 100 \mathrm{GeV}$. Then the smallness of neutrino masses is naturally explained by the so-called seesaw mechanism [11]. In this case, the right-handed sneutrino cannot be the LSP.

On the other hand, neutrinos can obtain very small masses without invoking the seesaw mechanism. By introducing right-handed (s)neutrinos with vanishing Majorana masses, neutrinos become massive Dirac fermions after the electroweak symmetry breaking. In this case, as we will show later, the neutrino Yukawa coupling constants are of the order of $\mathcal{O}\left(10^{-13}-10^{-12}\right)$ or smaller in order to account for the neutrino mass scales in the oscillation experiments. It should be noted that such small coupling constants are natural in the 'tHooft's sense [12]. This is owing to the fact that the chiral symmetry in neutrino sector is recovered in the limit of vanishing neutrino Yukawa coupling constants.

In supersymmetric theories, when neutrino masses are purely Dirac-type, right-handed sneutrinos receive masses dominantly from the effects of supersymmetry breaking. Throughout this paper, we consider gravity-mediation type models of supersymmetry breaking. Then, all the supersymmetry breaking masses are of the order of $0.1-1 \mathrm{TeV}$. As a result, there is a possibility that the lightest right-handed sneutrino $\tilde{\nu}_{R}$ is the LSP. In Ref. [3], we have shown that the LSP $\tilde{\nu}_{R}$ is a good candidate of CDM because it is stable and charge-neutral, and also because its relic density can become consistent with the dark matter density with relevant choice of parameters.

In the early universe, $\tilde{\nu}_{R}$ is not thermalized since its 
interaction is extremely weak. ${ }^{2}$ Thus, assuming that the initial abundance of $\tilde{\nu}_{R}$ is zero, ${ }^{3} \tilde{\nu}_{R}$ should be produced in some processes after inflation if $\tilde{\nu}_{R^{-}} \mathrm{CDM}$ is realized. One possible production is via decays of superparticles [3]. Superparticles in the minimal supersymmetric standard model (MSSM) are in chemical equilibrium when the temperature is high enough, so they are quite abundant in the early universe. Decays of those MSSM superparticles can produce $\tilde{\nu}_{R}$. The decay processes may occur when the MSSM superparticles are in the chemical equilibrium and when they freeze-out from the thermal bath. Importantly, the decay processes after the freeze-out may be important for the production of $\tilde{\nu}_{R}$. This happens for the decay of the lightest superparticle in the MSSM sector, which we call the MSSM-LSP. This is because the neutrino Yukawa coupling constants are so small that the lifetime of the NLSP becomes rather long. Such late decay of the NLSP into $\tilde{\nu}_{R}$ can be one important source of $\tilde{\nu}_{R^{-}} \mathrm{CDM}$.

In this paper, we consider the scenario with $\tilde{\nu}_{R}$-LSP and investigate its production in the early universe. In Ref. [3], we have mainly analyzed the production by decays of superparticles which are in chemical equilibrium. Here, we study another production process in detail, that is, decay of the MSSM-LSP after its freeze-out time. We estimate the relic density of $\tilde{\nu}_{R}$ from such a decay adopting the minimal supergravity model [13], and also discuss the implication of the $\tilde{\nu}_{R^{-}}$CDM scenario. We also reconsider the decay of MSSM superparticles in chemical equilibrium.

The outline of this article is the following. We start by reviewing the framework of our analysis in Sec. [II] where we present properties of neutrinos and sneutrinos under consideration. In Sec. III various production processes of $\tilde{\nu}_{R}$ are discussed. We first study the production of $\tilde{\nu}_{R}$ by decays of the NLSP after the freeze-out time. The constraint on the NLSP decay coming from the bigbang nucleosynthesis (BBN) is also discussed. We then turn to reconsider the production by decays of superparticle in chemical equilibrium to complete the discussion in Ref. [3]. In Sec. III, other possible mechanisms of the production of $\tilde{\nu}_{R}$ are also mentioned. Finally, we conclude in Sec. IV]

\section{FRAMEWORK}

First of all, let us explain the framework of our analysis. We consider the MSSM with three generations of right-handed (s)neutrinos, where neutrino masses are assumed to be purely Dirac-type. The superpotential is

\footnotetext{
2 If it was thermalized by some unknown interactions in the very beginnings of the universe, the present relic density of $\tilde{\nu}_{R}$ would overclose the universe and be inconsistent with the observation.

3 See, however, the discussion in Sec. IIID
}

then given by

$$
W=y_{\nu} \hat{H}_{u} \cdot \hat{L} \hat{\nu}_{R}^{c}-y_{e} \hat{H}_{d} \cdot \hat{L} \hat{\ell}_{R}^{c}+\mu_{H} \hat{H}_{d} \cdot \hat{H}_{u},
$$

where we have omitted terms with quark superfields since they are irrelevant for our discussion. Here $\hat{H}_{u}=$ $\left(\hat{H}_{u}^{+}, \hat{H}_{u}^{0}\right)$ and $\hat{H}_{d}=\left(\hat{H}_{d}^{0}, \hat{H}_{d}^{-}\right)$are the Higgs superfields coupled to up- and down-type quarks, and $\hat{L}=\left(\hat{\nu}_{L}, \hat{\ell}_{L}^{-}\right)$ are the left-handed lepton superfields. The superfields of right-handed neutrinos are denoted as $\hat{\nu}_{R}$. (In this article, "hat" is for superfields while "tilde" is for superparticles with odd $R$-parity.) $\mu_{H}$ is the supersymmetryinvariant Higgs mass. Here and hereafter, the generation indices are implicit for simplicity.

In this framework, neutrinos obtain masses as

$$
m_{\nu}=y_{\nu}\left\langle H_{u}^{0}\right\rangle=y_{\nu} v \sin \beta,
$$

where $v \simeq 174 \mathrm{GeV}$ is the vacuum expectation value (VEV) of the standard-model-like Higgs boson, and $\tan \beta=\left\langle H_{u}^{0}\right\rangle /\left\langle H_{d}^{0}\right\rangle$. Numerically, we obtain

$$
y_{\nu} \sin \beta \simeq 3.0 \times 10^{-13} \times\left(\frac{m_{\nu}^{2}}{2.8 \times 10^{-3} \mathrm{eV}^{2}}\right)^{1 / 2} .
$$

Importantly, neutrino-oscillation experiments have provided only the mass-squared differences of neutrinos 8 , 10]:

$$
\begin{aligned}
& {\left[\Delta m_{\nu}^{2}\right]_{\text {atom }} \simeq 2.8 \times 10^{-3} \mathrm{eV}^{2},} \\
& {\left[\Delta m_{\nu}^{2}\right]_{\text {solar }} \simeq 7.9 \times 10^{-5} \mathrm{eV}^{2},}
\end{aligned}
$$

and the absolute scales of neutrino masses have not been determined. There are then three possible mass spectra: (i) the normal hierarchy case, (ii) the inverted hierarchy case, and (iii) the degenerate case. On the other hand, the cosmological observations place the upper bound on $m_{\nu}$. Here, we adopt the upper bound derived from the WMAP three-year data: $\sum m_{\nu}<2.0 \mathrm{eV}(95 \% \mathrm{CL})$ [14], where summation is over all the three neutrino flavors. ${ }^{4}$ Then, we obtain

$$
m_{\nu}<0.67 \mathrm{eV}
$$

In the case with hierarchical neutrino masses (i) and (ii), the heaviest neutrino mass is well approximated by $\sqrt{\left[\Delta m_{\nu}^{2}\right]_{\text {atom }}}$ and the corresponding neutrino Yukawa coupling constant, which is the largest one, is about $y_{\nu} \sin \beta \simeq 3.0 \times 10^{-13}$. On the other hand, in the degenerate case (iii), the coupling constants become larger; if the neutrino masses take their largest possible value

4 The inclusion of the data other than the cosmic microwave background radiation (CMBR) observation (e.g., the large scale structure and the matter power spectrum inferred from the Ly$\alpha)$ makes the mass limit of neutrinos more stringent (see, for example, Ref. [1]). We take here a conservative approach and use the bound only from the CMBR data. 
of $\simeq 0.67 \mathrm{eV}$, Yukawa coupling constant is as large as $y_{\nu} \sin \beta \simeq 3.8 \times 10^{-12}$. We can see that the neutrino Yukawa coupling constants are very small in any case, which are much smaller than those of other quarks and leptons.

Next, we turn to discuss sneutrinos. For this purpose, let us introduce the soft supersymmetry breaking terms with sneutrinos as

$$
\begin{aligned}
-\mathcal{L}_{\text {soft }} \supset & \tilde{M}_{L}^{2}|\tilde{L}|^{2}+\tilde{M}_{\nu_{R}}^{2}\left|\tilde{\nu}_{R}\right|^{2} \\
& +\left(\tilde{A}_{\nu} H_{u} \cdot \tilde{L}_{\tilde{\nu}_{R}^{c}}^{c}-\tilde{A}_{e} H_{d} \cdot \tilde{L} \tilde{\ell}_{R}^{c}+\text { h.c. }\right)
\end{aligned}
$$

where $\tilde{M}_{L}, \tilde{M}_{\nu_{R}}$ and $\tilde{A}_{\nu}$ are supersymmetry breaking mass parameters. We parameterize $\tilde{A}_{\nu}$ as

$$
\tilde{A}_{\nu}=y_{\nu} A_{\nu}=y_{\nu} a_{\nu} \tilde{M}_{L},
$$

where $a_{\nu}$ is a dimension-less constant and $\left|a_{\nu}\right|$ is expected to be of the order of unity in simple models of supergravity. Due to the smallness of the neutrino Yukawa coupling constants, the left-right mixing of sneutrinos is very small. Thus, we can treat the left- and right-handed sneutrinos as mass eigenstates. Their masses are given by

$$
m_{\tilde{\nu}_{L}}^{2} \simeq \tilde{M}_{L}^{2}+\frac{1}{2} \cos 2 \beta m_{Z}^{2}, \quad m_{\tilde{\nu}_{R}}^{2} \simeq \tilde{M}_{\nu_{R}}^{2}
$$

where we have omitted the negligible contributions of $m_{\nu}^{2}$. The left-right mixing angle of sneutrinos is denoted by $\Theta$, which is found as

$$
\tan 2 \Theta=\frac{2 m_{\nu}\left|\cot \beta \mu_{H}-A_{\nu}^{*}\right|}{m_{\tilde{\nu}_{L}}^{2}-m_{\tilde{\nu}_{R}}^{2}},
$$

and $\Theta$ is highly suppressed because of the smallness of $m_{\nu}$. It can be seen from (10) that masses of right-handed sneutrinos are determined solely by the supersymmetry breaking masses, and hence the lightest right-handed sneutrino may become the LSP.

\section{PRODUCTION OF THE LSP RIGHT-HANDED SNEUTRINO}

As we have shown in Ref. [3], the LSP right-handed sneutrino is a viable candidate of CDM. This is because it is stable under the $R$-parity conservation, and also because it has only suppressed interactions, i.e., the neutrino Yukawa interactions and the gauge interactions through the left-right mixing.

In this section, we discuss production processes of right-handed sneutrino $\tilde{\nu}_{R}$ in the early universe and calculate the density parameter of right-handed sneutrino:

$$
\Omega_{\tilde{\nu}_{R}}=\frac{\rho_{\tilde{\nu}_{R}, 0}}{\rho_{\mathrm{cr}}},
$$

where $\rho_{\tilde{\nu}_{R}, 0}$ is the present energy density of $\tilde{\nu}_{R}$ and $\rho_{\text {cr }}=1.05 \times 10^{-5} h^{2} \mathrm{GeV} / \mathrm{cm}^{3}$ is the critical density. Importantly, there are two types of production processes of right-handed-sneutrino LSP; one is the decay of MSSM superparticles in the chemical equilibrium and the other is the decay of the MSSM-LSP after freeze out. We have considered the former effect in Ref. [3] and found that, in most of the cases, $\Omega_{\tilde{\nu}_{R}}$ via the decay of MSSM superparticles in the chemical equilibrium becomes smaller than $\Omega_{\mathrm{DM}}$. Thus, it is probable that the relic $\tilde{\nu}_{R}$ are mostly from the decay of the MSSM-LSP after freeze-out. So, we first consider the latter effect in detail although we will also discuss the former production process later.

\section{A. $\tilde{\nu}_{R}$ production via MSSM-LSP decay}

Let us discuss the production of $\tilde{\nu}_{R}$ via decays of the MSSM-LSP after freeze out. As we will discuss, the density parameter of $\tilde{\nu}_{R}$ is sensitive to the MSSM parameters. In this paper, we will not study the complete parameter space since it is beyond the scope of our purpose; we rather illustrate the basic features using a simple model of supersymmetry breaking. Here we consider, as an example, the minimal supergravity model with the gauge coupling unification at $M_{G} \simeq 2 \times 10^{16} \mathrm{GeV}$. This model is described by $m_{0}$ (the universal scalar mass at $M_{G}$ ), $m_{1 / 2}$ (the unified gaugino mass at $M_{G}$ ), and $\tilde{A}_{G}$ (the universal $A$-parameter at $M_{G}$ ) together with $\tan \beta$ and the sign of $\mu_{H}$. Then, the on-shell mass of $\tilde{\nu}_{R}$ is given by

$$
m_{\tilde{\nu}_{R}}=m_{0}
$$

since the renormalization group (RG) evolution of $m_{\tilde{\nu}_{R}}$ is negligible due to the smallness of $y_{\nu}$.

Adopting the minimal supergravity model, three righthanded sneutrinos are almost degenerate. The mass differences are mainly from the effects of RG evolution through $y_{\nu}$, and hence they are very small. ${ }^{5}$ Consequently, all the three right-handed sneutrinos are stable within the age of the universe and contribute to the darkmatter density.

Since the neutrino Yukawa coupling constants are negligibly small, the decay of the MSSM-LSP into $\tilde{\nu}_{R}$ is extremely suppressed. Detailed value of the lifetime of the MSSM-LSP depends on MSSM parameters. However, as we will show later, lifetime of the MSSM-LSP is long enough so that the decay of the MSSM-LSP occurs sufficiently after its freeze out.

The density parameter of $\tilde{\nu}_{R}$ from the decay of the MSSM-LSP after freeze out is given by

$$
\Omega_{\tilde{\nu}_{R}}^{\mathrm{FO}}=\frac{m_{\tilde{\nu}_{R}}}{m_{\mathrm{MSSM}-\mathrm{LSP}}} \Omega_{\mathrm{MSSM}-\mathrm{LSP}},
$$

where $\Omega_{\text {MSSM-LSP }}$ is the (would-be) present density parameter of the MSSM-LSP for the case where it is stable.

\footnotetext{
5 Other sources of the mass differences are the neutrino masses and the contributions from the left-right mixing of sneutrinos.
} 

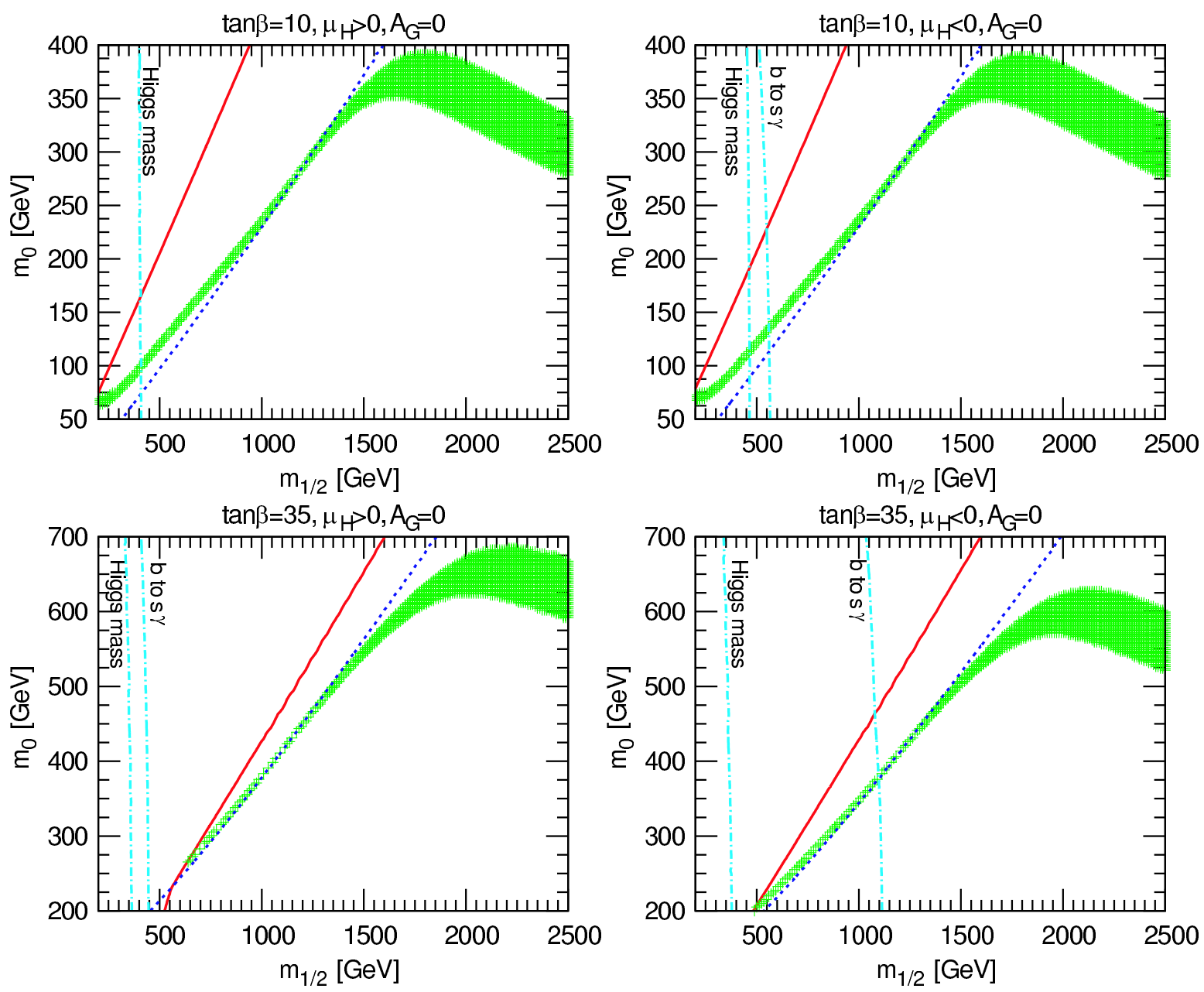

FIG. 1: The parameter range to realize $\tilde{\nu}_{R}$-CDM in the minimal supergravity model with $A_{G}=0$ (shown by green "+" marks). The solid line represents $m_{\tilde{\nu}_{R}}=m_{\mathrm{MSSM}-\mathrm{LSP}} ; \tilde{\nu}_{R}$ becomes the LSP below this line. The MSSM-LSP is the lightest neutralino $\tilde{\chi}_{1}^{0}$ or the lighter stau $\tilde{\tau}_{1}$ in the region above or below the dashed line. The vertical dot-dashed lines show the lower bounds on $m_{1 / 2}$ from the Higgs mass and $b \rightarrow s \gamma$.

$\Omega_{\mathrm{MSSM}-\mathrm{LSP}}$ is estimated using conventional method since the neutrino Yukawa coupling constants are very small. ${ }^{6}$ $\tilde{\nu}_{R}$ can account for the present dark-matter density if $\Omega_{\tilde{\nu}_{R}}^{\mathrm{FO}}=\Omega_{\mathrm{DM}}$. We note here that $\Omega_{\tilde{\nu}_{R}}^{\mathrm{FO}}$ is insensitive to the reheating temperature $T_{R}$ after inflation as long as $T_{R}$ is higher than $T_{F}$, where $T_{F}$ is the freeze-out temperature which is roughly given by $T_{F} \sim m_{\text {MSSM-LSP }} / 20$.

Based on Eq. (14), $\tilde{\nu}_{R}$-CDM is realized with some choice of MSSM parameters which satisfies the following relation:

$$
\Omega_{\mathrm{MSSM}-\mathrm{LSP}}=\frac{m_{\mathrm{MSSM}-\mathrm{LSP}}}{m_{\tilde{\nu}_{R}}} \Omega_{\mathrm{DM}} .
$$

6 In this analysis, we assume that only the thermal relic of the MSSM-LSP contributes to $\Omega_{\text {MSSM-LSP. The MSSM-LSP might }}$ be also produced via non-thermal processes [15]. Even in this case, if $\Omega_{\text {MSSM-LSP }}$ is given as (15), the $\tilde{\nu}_{R}$-CDM is realized.
Since $m_{\text {MSSM-LSP }} \neq m_{\tilde{\nu}_{R}}$, this implies that the MSSM parameters realizing $\tilde{\nu}_{R}$-CDM are different from those for the conventional scenario where the MSSM-LSP, say the lightest neutralino, becomes CDM. Furthermore, $\tilde{\nu}_{R^{-}}$ CDM is possible even if the MSSM-LSP is an electrically charged and/or colored superparticle, and hence the $\tilde{\nu}_{R^{-}}$ $\mathrm{CDM}$ is realized in a wider parameter range compared with the MSSM-LSP dark matter.

In the parameter range of interest, the MSSM-LSP is the lightest neutralino $\tilde{\chi}_{1}^{0}$ which is Bino-like or the lighter stau $\tilde{\tau}_{1}$. We estimate their (would-be) relic density $\Omega_{\mathrm{MSSM}-\mathrm{LSP}} h^{2}$ by using the micrOMEGAs package [16], and then calculate $\Omega_{\tilde{\nu}_{R}}^{\mathrm{FO}} h^{2}$ using Eq. (14). In Fig. 1, we show the parameter region where $\Omega_{\tilde{\nu}_{R}}^{\mathrm{FO}} h^{2}$ is in the range (11). Here, for simplicity, we set $A_{G}=0$. In the figure, we also impose the following phenomenological constraints; the mass of the lighter neutral Higgs boson (the standardmodel like Higgs boson) be larger than the experimental 


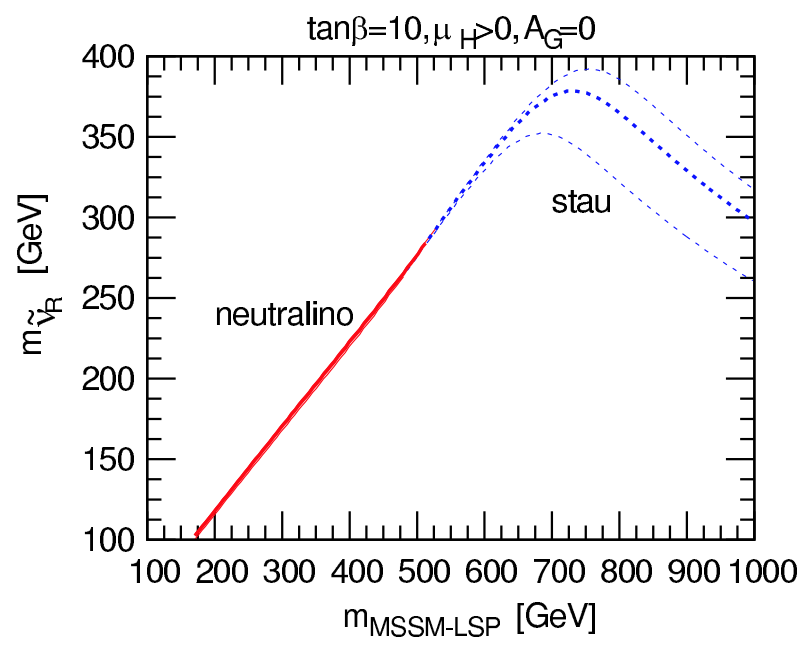

FIG. 2: The mass of $\tilde{\nu}_{R}$-CDM in the minimal supergravity model. We take here $A_{G}=0, \tan \beta=10$ and $\mu_{H}>0$. The thick line corresponds to $\Omega_{\tilde{\nu}_{R}}^{\mathrm{FO}} h^{2}=0.105$, and the thin lines correspond to $\Omega_{\tilde{\nu}_{R}}^{\mathrm{FO}} h^{2}$ in the range (1). The solid or dashed lines are for the $\tilde{\chi}_{1}^{0}$ or $\tilde{\tau}_{1}$ MSSM-LSP, respectively.

bound [17]

$$
m_{h^{0}}>114.4 \mathrm{GeV}
$$

and the branching ratio of $b \rightarrow s \gamma$ be in the three-sigma range of the observational data [18]:

$$
\operatorname{Br}(b \rightarrow s \gamma)=(3.55 \pm 0.78) \times 10^{-4} .
$$

One can see that $\tilde{\nu}_{R}$ can be CDM of the universe in a wide parameter region. In addition, we emphasize that $\tilde{\nu}_{R^{-}}$CDM can be realized not only in the case where $\tilde{\chi}_{1}^{0}$ is the MSSM-LSP but also in the case with $\tilde{\tau}_{1}$-MSSM-LSP. This is an interesting feature of the present scenario. We also stress here that $\Omega_{\tilde{\nu}_{R}}^{\mathrm{FO}}$ is insensitive to the neutrino Yukawa coupling constants.

In Fig. 2, we show the mass of right-handed sneutrino which gives the correct value of $\Omega_{\tilde{\nu}_{R}}^{\mathrm{FO}}$ to realize $\tilde{\nu}_{R}$-CDM as a function of the mass of the MSSM-LSP. Here, we take $A_{G}=0, \tan \beta=10$ and $\mu_{H}>0$; in this case, when $\Omega_{\tilde{\nu}_{R}}^{\mathrm{FO}} h^{2}=0.105$, the MSSM-LSP is the lightest neutralino for $m_{\text {MSSM-LSP }} \lesssim 510 \mathrm{GeV}$, and is stau for $m_{\text {MSSM-LSP }} \gtrsim$ $510 \mathrm{GeV}$. Fig. 2 shows that the mass of the right-handed sneutrino is bounded from above to realize $\tilde{\nu}_{R}$-CDM.

In the region where $\tilde{\chi}_{1}^{0}$ becomes the MSSM-LSP, we find an approximate relation $m_{\tilde{\chi}_{1}^{0}} \simeq 1.8 m_{\tilde{\nu}_{R}}$ to realize $\Omega_{\tilde{\nu}_{R}}^{\mathrm{FO}}=\Omega_{\mathrm{DM}}$. In this case, the mass of the $\tilde{\nu}_{R^{-}} \mathrm{CDM}$ is well determined from $m_{\tilde{\chi}_{1}^{0}}$ even if one varies $\Omega_{\tilde{\nu}_{R}}^{\mathrm{FO}} h^{2}$ in the range given in Eq. (11). Moreover, we find that masses of $\tilde{\chi}_{1}^{0}$ and $\tilde{\tau}_{1}$ are close to each other and their co-annihilation effects is important in estimating $\Omega_{\text {MSSM-LSP }}$. (See the following discussion and Fig. (4))

As $m_{\tilde{\chi}_{1}^{0}}$ becomes larger, the MSSM-LSP is $\tilde{\tau}_{1}$. Then, the relation $\Omega_{\tilde{\nu}_{R}}^{\mathrm{FO}}=\Omega_{\mathrm{DM}}$ is realized even when $m_{\tilde{\chi}_{1}^{0}}$ is much larger than $\sim 1 \mathrm{TeV}$. For $m_{\tilde{\chi}_{1}^{0}} \gg 700 \mathrm{GeV}$, the yield variable of $\tilde{\tau}_{1}$, which is defined by the ratio of the number density to the entropy density, is given by [19]

$$
Y_{\tilde{\tau}_{1}} \simeq 10^{-12} \times c_{Y}\left(\frac{m_{\tilde{\tau}_{1}}}{1 \mathrm{TeV}}\right)
$$

where $c_{Y}$ is a constant of the order of unity; we numerically found that $c_{Y}=1.2-1.3$ in the parameter region we are interested in. We can then obtain the relic density of $\tilde{\nu}_{R}$ from $\tilde{\tau}_{1}$ decay as

$$
\Omega_{\tilde{\nu}_{R}}^{\mathrm{FO}} h^{2} \simeq 0.027 \times c_{Y}\left(\frac{m_{\tilde{\tau}_{1}}}{1 \mathrm{TeV}}\right)\left(\frac{m_{\tilde{\nu}_{R}}}{100 \mathrm{GeV}}\right) .
$$

The numerical calculation shows that, when $m_{\tilde{\nu}_{R}}=m_{0}=$ $100 \mathrm{GeV}$, for example, $\Omega_{\tilde{\nu}_{R}}^{\mathrm{FO}} h^{2}=0.105$ is realized for $m_{\tilde{\tau}_{1}} \simeq 3.1 \mathrm{TeV}$, which corresponds to $m_{1 / 2} \simeq 8.7 \mathrm{TeV}$ and $m_{\tilde{\chi}_{1}^{0}} \simeq 4.1 \mathrm{TeV}$. Even when we take larger value of $m_{\tilde{\tau}_{1}}$ (and $m_{\tilde{\chi}_{1}^{0}}$ ), the $\tilde{\nu}_{R^{-}} \mathrm{CDM}$ is possible by taking $m_{\tilde{\nu}_{R}} \lesssim 100 \mathrm{GeV}$, as seen from Eq. (19).

Now, we come to a position to discuss the lifetime of the MSSM-LSP. As we mentioned, Eq. (14) is applicable when the MSSM-LSPs decay after the time of freeze out. In order to see if this is the case, let us estimate the decay rate of the MSSM-LSP. When the MSSM-LSP is the Bino-like neutralino, it decays into $\tilde{\nu}_{R}$ and anti-neutrino (and also its CP conjugate state) through the left-right mixing of sneutrinos. The decay rate is estimated as

$$
\begin{aligned}
\Gamma_{\tilde{\chi}_{1}^{0}} & \simeq \Gamma_{\tilde{B} \rightarrow \tilde{\nu}_{R} \bar{\nu}}+\Gamma_{\tilde{B} \rightarrow \tilde{\nu}_{R}^{c} \nu} \\
& =\frac{g_{1}^{2} \Theta^{2}}{32 \pi} m_{\tilde{\chi}_{1}^{0}}\left(1-\frac{m_{\tilde{\nu}_{R}}^{2}}{m_{\tilde{\chi}_{1}^{0}}^{2}}\right)^{2},
\end{aligned}
$$

where $g_{1}$ is a gauge coupling constant of $\mathrm{U}(1)_{Y}$. Notice that the Bino-like neutralino $\tilde{\chi}_{1}^{0}$ universally couples to (s)neutrinos in all three generations. Thus, the decay process into the sneutrino which interacts via the strongest Yukawa interaction is the most important since the mixing angle $\Theta$ is proportional to the Yukawa coupling constant.

When the MSSM-LSP is $\tilde{\tau}_{1}$, the situation is slightly more complicated since the dominant decay mode depends on the mass difference between $\tilde{\tau}_{1}$ and $\tilde{\nu}$. When $m_{\tilde{\tau}_{1}}>m_{\tilde{\nu}_{R}}+m_{W}$, the decay rate is given by

$$
\begin{aligned}
\Gamma_{\tilde{\tau}_{1}} \simeq & \Gamma_{\tilde{\tau}_{1} \rightarrow \tilde{\nu}_{R} W} \\
= & \frac{g_{2}^{2} \Theta^{2}}{32 \pi}\left|U_{L 1}^{\left(\tilde{\tau}_{1}\right)}\right|^{2}\left|U_{\tau 3}^{\mathrm{MNS}}\right|^{2} \frac{m_{\tilde{\tau}_{1}}^{3}}{m_{W}^{2}} \\
& \times\left[1-\frac{2\left(m_{\tilde{\nu}_{R}}^{2}+m_{W}^{2}\right)}{m_{\tilde{\tau}_{1}}^{2}}+\frac{\left(m_{\tilde{\nu}_{R}}^{2}-m_{W}^{2}\right)^{2}}{m_{\tilde{\tau}_{1}}^{4}}\right]^{3 / 2},
\end{aligned}
$$

where $g_{2}$ is a gauge coupling constant of $\mathrm{SU}(2)_{L}, m_{W}$ is the $W$-boson mass, and $U^{\left(\tilde{\tau}_{1}\right)}$ is the mixing matrix of staus which relates the gauge eigenstates and mass eigenstates as

$$
\left(\begin{array}{c}
\tilde{\tau}_{L} \\
\tilde{\tau}_{R}
\end{array}\right)=U^{(\tilde{\tau})}\left(\begin{array}{c}
\tilde{\tau}_{1} \\
\tilde{\tau}_{2}
\end{array}\right)
$$




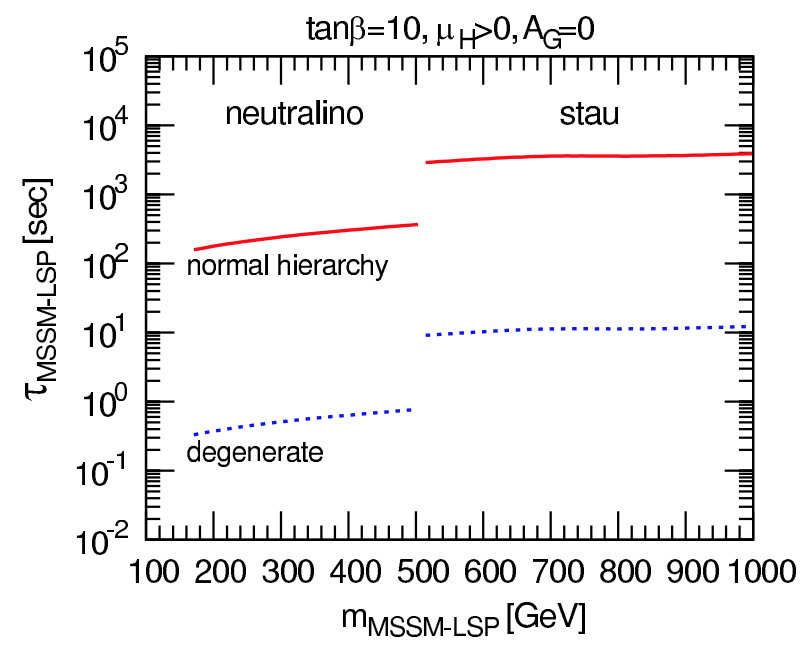

FIG. 3: The lifetime of the MSSM-LSP yielding $\Omega_{\tilde{\nu}_{R}}^{\mathrm{FO}} h^{2}=$ 0.105 in the minimal supergravity model. We take here $A_{G}=$ $0, \tan \beta=10$ and $\mu_{H}>0$. The MSSM-LSP is $\tilde{\chi}_{1}^{0}$ or $\tilde{\tau}_{1}$ when $m_{\text {MSSM-LSP }}$ is smaller or larger than about $510 \mathrm{GeV}$. The solid lines are the lifetime assuming the normal hierarchy of neutrino masses and taking $m_{\nu}=\sqrt{\left[\Delta m_{\nu}^{2}\right]_{\text {atom }}}$, while the dashed lines are the lifetime assuming the degenerate neutrino masses and taking $m_{\nu}=0.67 \mathrm{eV}$.

where $\tilde{\tau}_{1}$ and $\tilde{\tau}_{2}$ are mass eigenstates with $m_{\tilde{\tau}_{1}} \leq m_{\tilde{\tau}_{2}}$. In addition, $U^{\mathrm{MNS}}$ is the neutrino mixing matrix. Here, we use $U_{\tau 3}^{\mathrm{MNS}}=1 / \sqrt{2}$. Notice that the above expression is relevant for the case with hierarchical neutrino mass; for the degenerate case, decay processes into all three sneutrinos may be equally important. For example, when the mass differences among three left-handed sleptons are negligible, $\left|U_{\tau 3}^{\mathrm{MNS}}\right|^{2}$ should be replaced by 1. Note that $\tilde{\tau}_{1}$ may also decay into $\tilde{\nu}_{R}$ and the charged Higgs boson. Such a decay channel is, however, kinematically forbidden in the parameter region which we are interested in. When the decay process $\tilde{\tau}_{1} \rightarrow \tilde{\nu}_{R} W$ is kinematically forbidden, $\tilde{\tau}_{1}$ mainly decays as $\tilde{\tau}_{1} \rightarrow \tilde{\nu}_{R} \ell \bar{\nu}$, $\tilde{\nu}_{R} q \bar{q}^{\prime}$ with exchanging virtual $W$-boson.

Using $y_{\nu} \simeq 3.0 \times 10^{-13}$, which is the suggested value of the neutrino Yukawa coupling constant in the case with the normal hierarchy neutrino mass matrix, the lifetime of the MSSM-LSP for the case yielding $\Omega_{\tilde{\nu}_{R}}^{\mathrm{FO}} h^{2}=0.105$ is shown in Fig. 3. Here, we take $A_{G}=0$ to illustrate the typical value of the lifetime. We can see that the lifetime is roughly of the order of $10^{2}-10^{3} \mathrm{sec}$, and hence the decay of the MSSM-LSP occurs after its freeze-out time. When neutrino masses are degenerate, the lifetime of the MSSM-LSP becomes significantly shorter because the neutrino Yukawa coupling constants are enhanced and also because the MSSM-LSP decays into three (almost) degenerate right-handed sneutrinos. Even in this case, the MSSM-LSP decays into $\tilde{\nu}_{R}$ after the time of the freeze-out. Therefore, calculation with Eq. (14) is justified with any of the neutrino mass matrix.

Here, we should comment on the fact that the lifetime of the MSSM-LSP depends on the $A$-parameter since the left-right mixing angle of the neutrino $\Theta$ is sensitive to it. By solving RG equations, we found that $A_{\nu}$ parameter at the scale $m_{Z}$ is given by

$$
A_{\nu} \simeq A_{G}-0.59 m_{1 / 2} .
$$

Thus, as $A_{G}$ changes, the lifetime of the MSSM-LSP varies.

We would like to note that the $\tilde{\nu}_{R^{-}} \mathrm{CDM}$ scenario and MSSM-LSP dark matter scenario are realized in different parameter space. In particular, we emphasize that $\Omega_{\mathrm{MSSM}-\mathrm{LSP}}>\Omega_{\mathrm{DM}}$ is required if the right-handed sneutrino produced from the decay of MSSM-LSP after freeze out becomes CDM. Using this fact, we can potentially distinguish the scenario of $\tilde{\nu}_{R^{-}} \mathrm{CDM}$ and MSSM-LSP dark matter with precise determinations of the properties of MSSM particles by future collider experiments. To see this, in Fig. 目, we show the regions on $m_{\tilde{\chi}_{1}^{0}}$ vs. $m_{\tilde{\tau}_{1}}-m_{\tilde{\chi}_{1}^{0}}$ plane where the density parameter of the LSP becomes consistent with the presently observed dark matter density for the cases where the LSP is $\tilde{\nu}_{R}$ and $\tilde{\chi}_{1}^{0}$. We can see that, for a given value of $m_{\tilde{\chi}_{1}^{0}}$, the suggested values of $m_{\tilde{\tau}_{1}}$ are different by $5 \mathrm{GeV}$ or so between two scenarios. We also show the correlations between $m_{\tilde{\tau}_{1}}$ and $m_{\tilde{\chi}_{1}^{+}}$ (with $\tilde{\chi}_{1}^{+}$being the lighter chargino) in two scenarios. We can see that, for a given $m_{\tilde{\tau}_{1}}$, the suggested value of $m_{\tilde{\chi}_{1}^{+}}$is different by $\sim 10 \mathrm{GeV}$. Thus, with the precise measurements of the masses of MSSM superparticles by future collider experiments, like the LHC and ILC, we may be able to distinguish the $\tilde{\nu}_{R}$-CDM and the MSSMLSP-CDM scenarios.

\section{B. Constraints from BBN}

As one can see from Fig. 3, the lifetime of the MSSMLSP may be so long that the decay of the MSSM-LSP occurs around or after the epoch of BBN. If so, abundances of light elements which are produced by the standard BBN reactions are affected by the decay of the MSSM-LSP and, consequently, the success of BBN may be spoiled.

Effects of late-decaying particles on BBN are intensively studied in Ref. [20]; the most important effects of the late-decaying particles are from $p \leftrightarrow n$ conversion, hadro-dissociation, and/or photo-dissociation process, depending on the lifetime. For the case of our interest, lifetime of the MSSM-LSP is $\lesssim 10^{4}$ sec. In such a case, $p \leftrightarrow n$ conversion and hadro-dissociation are important. If the abundance of the MSSM-LSP is too large, the abundances of light elements are too much affected to be consistent with observations by these processes. In order not to spoil the success of BBN, upper bound on the combination $B_{\text {had }} E_{\mathrm{vis}} Y_{\mathrm{MSSM}-\mathrm{LSP}}$ is obtained. In our case, $B_{\text {had }}$ is the hadronic branching ratio of the MSSMLSP, $E_{\mathrm{vis}}$ is the net energy carried away by hadrons in the decay of the MSSM-LSP, and $Y_{\text {MSSM-LSP }}$ is the yield 

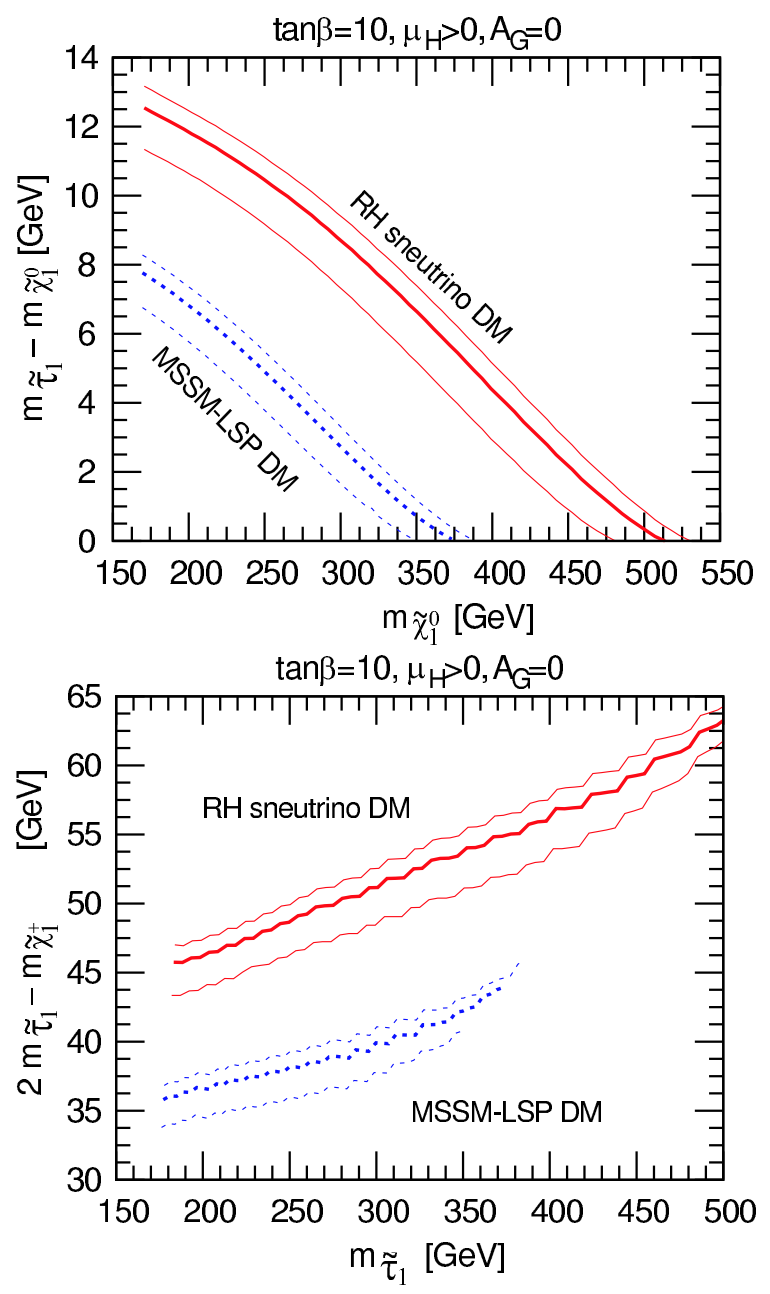

FIG. 4: The mass differences $m_{\tilde{\tau}_{1}}-m_{\tilde{\chi}_{1}^{0}}$ and $2 m_{\tilde{\tau}_{1}}-m_{\tilde{\chi}_{1}^{+}}$ realizing $\tilde{\nu}_{R}$-CDM in the minimal supergravity model. We take here $A_{G}=0, \tan \beta=10$ and $\mu_{H}>0$. The thick solid line corresponds to $\Omega_{\tilde{\nu}_{R}}^{\mathrm{FO}} h^{2}=0.105$ and the thin solid lines correspond to $\Omega_{\tilde{\nu}_{R}}^{\mathrm{FO}} h^{2}$ in the range (1). For comparison, we also show the mass differences in the case where the MSSMLSP $\tilde{\chi}_{1}^{0}$ becomes dark matter by dashed lines.

of the MSSM-LSP. In order to see if $\tilde{\nu}_{R^{-}}$CDM is viable, we will calculate these quantities in the following.

We first consider the case where the MSSM-LSP is $\tilde{\chi}_{1}^{0}$. Even though the dominant decay mode of $\tilde{\chi}_{1}^{0}$ is into neutrino and right-handed sneutrino, there still exist hadronic decay modes:

$$
\tilde{\chi}_{1}^{0} \rightarrow \tilde{\nu}_{R} \bar{\nu} q \bar{q}, \tilde{\nu}_{R}^{*} \nu q \bar{q}, \tilde{\nu}_{R} \ell^{+} q \bar{q}^{\prime}, \tilde{\nu}_{R}^{*} \ell^{-} q \bar{q}^{\prime},
$$

where $q$ and $q^{\prime}$ are quarks and $\ell^{ \pm}$are charged leptons. These processes are mediated by on-shell and/or off-shell gauge bosons. We have numerically calculated the decay rate of these processes and obtained $B_{\mathrm{had}}$ and $E_{\mathrm{vis}}$. Results for the case with $A_{G}=0, \tan \beta=10$ and $\mu_{H}>0$ are shown in Fig. 5. As one can see, hadronic branching ratio becomes $O\left(10^{-4}-10^{-3}\right)$ when the mass of $\tilde{\chi}_{1}^{0}$ is relatively large. This is because, when $m_{\tilde{\chi}_{1}^{0}}$ is large enough,
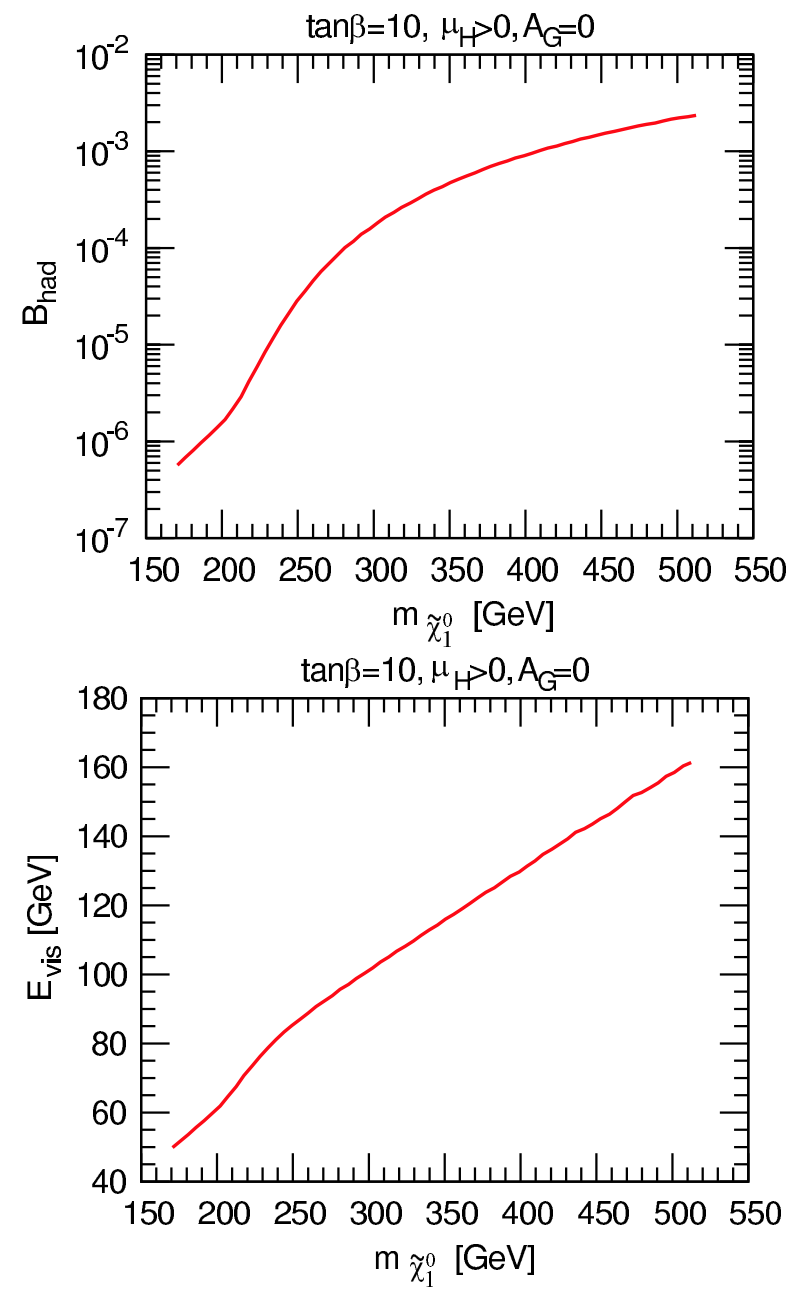

FIG. 5: The hadronic branching ratio $B_{\text {had }}$ and the net energy carried by hadrons $E_{\mathrm{vis}}$ in the $\tilde{\chi}_{1}^{0}$ MSSM-LSP decay. Here we consider the minimal supergravity model with $A_{G}=0$, $\tan \beta=10$ and $\mu_{H}>0$, and require $\Omega_{\tilde{\nu}_{R}}^{\mathrm{FO}} h^{2}=0.105$.

the decay modes $\tilde{\chi}_{1}^{0} \rightarrow \nu_{R} l^{+} W^{-}$and $\tilde{\chi}_{1}^{0} \rightarrow \nu_{R} \bar{\nu} Z$ (and their CP conjugated processes) are kinematically allowed and hence hadrons can be produced via the "three-body" decay processes. On the contrary, when $m_{\tilde{\chi}_{1}^{0}}$ is small, the weak bosons are always off-shell and $B_{\text {had }}$ is suppressed.

For MSSM parameters which give correct value of $\Omega_{\tilde{\nu}_{R}}^{\mathrm{FO}}$ to realize $\tilde{\nu}_{R}$-CDM, we calculate the product $B_{\text {had }} E_{\mathrm{vis}} Y_{\mathrm{MSSM}-\mathrm{LSP}}$ as a function of the MSSM-LSP mass. The results are shown in Fig. 6. For the same parameter set, we also calculate the lifetime of the MSSM-LSP. Once the lifetime of the MSSM-LSP is given, we can estimate upper bound on the product $B_{\mathrm{had}} E_{\mathrm{vis}} Y_{\mathrm{MSSM} \text {-LSP }}$ in order not spoil the success of BBN. When the lifetime is longer than $\sim 100 \mathrm{sec}$, the most important bounds come from the abundances of $\mathrm{D} / \mathrm{H}$ and ${ }^{6} \mathrm{Li} / \mathrm{H}$; here we adopt constraints, which are conservative ones, based on Fig. 39 


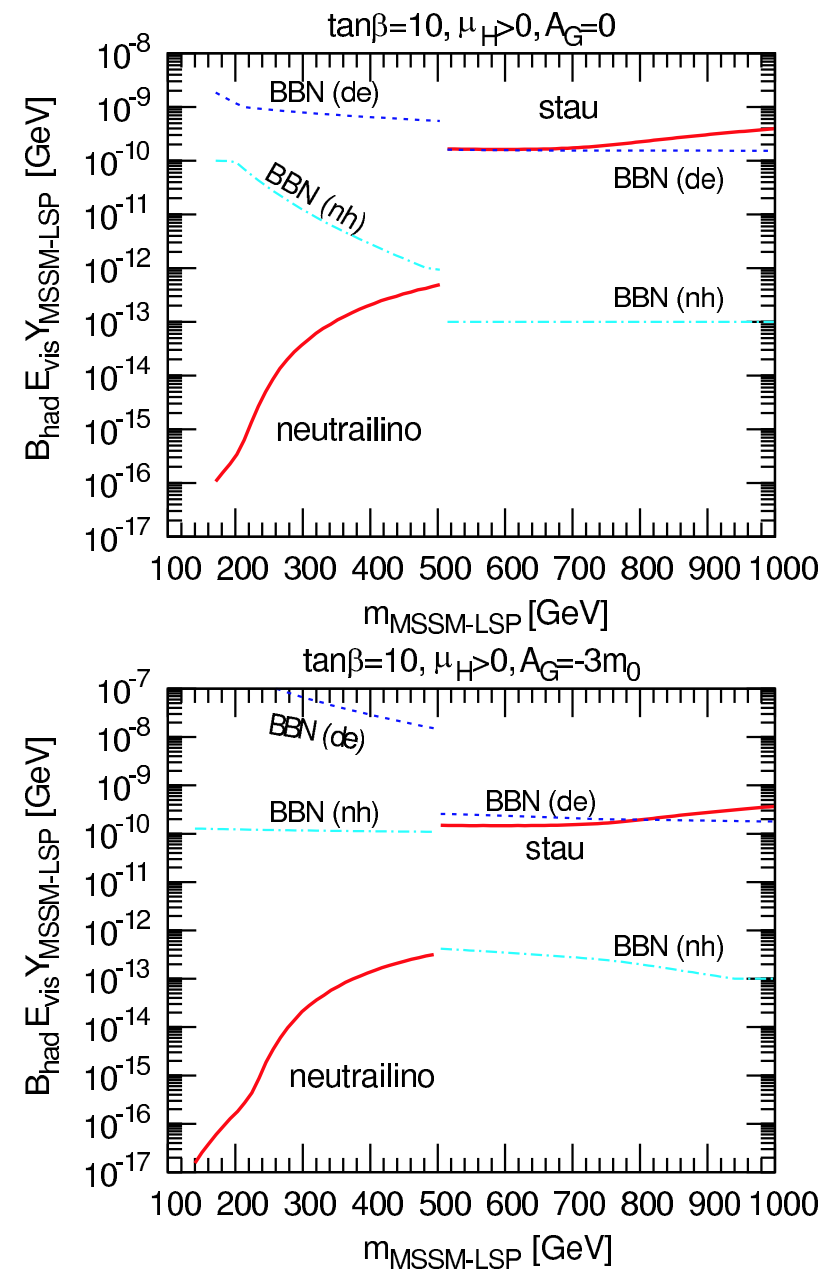

FIG. 6: The product $B_{\text {had }} E_{\text {vis }} Y_{\text {MSSM-LSP }}$ in terms of the MSSM-LSP mass in the minimal supergravity model. (shown by the solid lines). The upper or lower panel is for the case when $A_{G}=0$ or $A_{G}=-3 m_{0}$, respectively. Here we take $\tan \beta=10$ and $\mu_{H}>0$, and require $\Omega_{\tilde{\nu}_{R}}^{\mathrm{FO}} h^{2}=0.105$. The MSSM-LSP is $\tilde{\chi}_{1}^{0}$ or $\tilde{\tau}_{1}$ for $m_{\text {MSSM-LSP is greater or smaller }}$ than about $510 \mathrm{GeV}$. The dot-dashed lines show the upper bound from the BBN constraint on the MSSM-LSP decay by assuming the normal hierarchy of neutrino masses and taking $m_{\nu}=\sqrt{\left[\Delta m_{\nu}^{2}\right]_{\text {atom }}}$. The dashed lines show the bound by assuming the degenerate neutrino masses and taking $m_{\nu}=0.67$ $\mathrm{eV}$.

in Ref. [20]:

$$
y< \begin{cases}-13 & \text { for } 12>x>3.1 \\ -1.8 x-7.5 & \text { for } 3.1>x>2.6 \\ -6.6 x+4.8 & \text { for } 2.6>x>1.6\end{cases}
$$

where $x$ and $y$ are

$$
\begin{aligned}
& x=\log _{10}\left(\tau_{\text {MSSM-LSP }} / \mathrm{sec}\right), \\
& y=\log _{10}\left(B_{\text {had }} E_{\text {vis }} Y_{\text {MSSM-LSP }} / \mathrm{GeV}\right) .
\end{aligned}
$$

With shorter lifetime, overproduction of ${ }^{4} \mathrm{He}$ gives an upper bound on the abundance of the MSSM-LSP; here we show the constraint based on the observational constraint given in Ref. [21]:

$$
y< \begin{cases}-0.17 x-9.6 & \text { for } 3.5>x>0.39 \\ -0.88 x-9.4 & \text { for } 0.39>x>-0.41 \\ -3.8 x-10.6 & \text { for } \quad-0.41>x>-1.2\end{cases}
$$

Upper bound on $B_{\text {had }} E_{\mathrm{vis}} Y_{\text {MSSM-LSP }}$ is also shown in Fig. 6. Notice that the lifetime of MSSM-LSP depends on the MSSM parameters and also on the neutrino Yukawa coupling constants, so we show the upper bound for several cases.

With hierarchical neutrino mass matrix, we can see that $\tilde{\nu}_{R^{-}} \mathrm{CDM}$ is viable in most of the cases, although some parameter region with large $m_{\tilde{\chi}_{1}^{0}}$ may be constrained from the BBN, in particular for the case with $A_{G}=0$. If we consider the degenerate case with $m_{\nu}=0.67 \mathrm{eV}$, lifetime of $\tilde{\chi}_{1}^{0}$ becomes much shorter and hence the bound becomes weaker. Therefore, $\tilde{\nu}_{R}$-CDM does not conflict with the BBN constraints irrespective of the value of $A_{G}$, as long as there is no cancellation in the low-energy value of $A_{\nu}$ (see Eq. (23)).

Next, we consider the case where the MSSM-LSP is $\tilde{\tau}_{1}$. As mentioned before, when $\tilde{\tau}_{1}$ is the MSSM-LSP, $\tilde{\nu}_{R}$-CDM is realized when $m_{\tilde{\tau}_{1}}$ is relatively large. In this case, $\tilde{\tau}_{1}$ mainly decays into $\tilde{\nu}_{R}$ and $W$. Since $W$-boson decays into quarks with the branching ratio of $2 / 3$, we estimate the hadronic branching ratio of $\tilde{\tau}_{1}$ as

$$
B_{\text {had }}=\frac{2}{3} .
$$

In addition, $E_{\mathrm{vis}}$ is given by

$$
E_{\mathrm{vis}}=\frac{m_{\tilde{\tau}_{1}}^{2}+m_{W}^{2}-m_{\tilde{\nu}_{R}}^{2}}{2 m_{\tilde{\tau}_{1}}} .
$$

In Fig. 6, we also plot $B_{\text {had }} E_{\text {vis }} Y_{\text {MSSM-LSP }}$ as a function of the mass of $\tilde{\tau}_{1}$. Compared to the case with $\tilde{\chi}_{1}^{0}$-MSSMLSP, $B_{\text {had }} E_{\text {vis }} Y_{\text {MSSM-LSP }}$ becomes much larger since the hadronic branching ratio is close to 1 in this case. In particular, with the normal hierarchy of the neutrino masses, lifetime of $\tilde{\tau}_{1}$ is of the order of $10^{3} \mathrm{sec}$ or longer as far as $\left|a_{\nu}\right| \sim O(1)$. In this case, the whole region of the $\tilde{\tau}_{1}$ MSSM-LSP yielding $\Omega_{\tilde{\nu}_{R}}^{\mathrm{FO}}=\Omega_{\mathrm{DM}}$ is excluded. Notice that, although we have shown only the results when $m_{\tilde{\tau}_{1}} \lesssim 1 \mathrm{TeV}$, the $\tilde{\tau}_{1}$ MSSM-LSP with $m_{\tilde{\tau}_{1}} \gg 1 \mathrm{TeV}$ is also excluded since the lifetime and $E_{\mathrm{vis}}$ become larger.

On the other hand, for the case of degenerate neutrino masses, there appear the regions in which the $\tilde{\tau}_{1}$ MSSMLSP is consistent with the BBN. In such regions, the lifetime of $\tilde{\tau}_{1}$ is sufficiently short and it only receives the weaker constraint (27). We find that the possible masses of $\tilde{\tau}_{1}$ are relatively small and $m_{\tilde{\tau}_{1}} \gtrsim 1 \mathrm{TeV}$ is excluded by the BBN even with degenerate neutrinos.

We also find that the light $\tilde{\tau}_{1}$ is also excluded by the BBN when $\tan \beta$ is large. This is because, in such a case, the mass difference between $\tilde{\nu}_{R}$ and $\tilde{\tau}_{1}$ is smaller than $m_{W}$, and the decay channel $\tilde{\tau}_{1} \rightarrow \tilde{\nu}_{R}+W$ is kinematically 
blocked. If this is the case, the lifetime becomes longer and more stringent constraints apply.

From Fig. 6, we can learn that BBN constraint puts the upper bound on the mass of the MSSM-LSP. Interestingly, this bound excludes the possibility of the MSSMLSP being very heavy if $\tilde{\nu}_{R}$ is CDM, say being heavier than of the order of $\mathrm{TeV}$.

Before closing this subsection, we should comment that some of the results obtained in the previous and this subsections are from the fact that we use the minimal supergravity model. In particular, in the minimal supergravity model, masses of right-handed sneutrinos are equal to $m_{0}$. In this case, we obtained upper bound on the mass of the MSSM-LSP if $\tilde{\nu}_{R}$ is CDM. This result may not hold if we consider other types of models of supersymmetry breaking. In particular, if we adopt smaller sneutrino masses, we can push up the mass of the MSSMLSP. Furthermore, if the mass of the left-handed sneutrino becomes close to $m_{\tilde{\nu}_{R}}$, the left-right mixing angle of sneutrinos are enhanced. (See Eq. (11).) In this case, the lifetime of the MSSM-LSP becomes shorter and, as a results, the BBN constraint becomes weaker. In such a situation, the $\tilde{\tau}_{1}$ MSSM-LSP is possible in a wider parameter space. Finally, we should mention that the MSSMLSP can be a superparticle other than the Bino-like neutralino or the lighter stau. To realize the $\tilde{\nu}_{R} \mathrm{CDM}$, the MSSM-LSP may be electrically charged or colored superparticle, if its relic abundance satisfies (15) and if its decay is consistent with the BBN.

\section{Decays of superparticles in chemical equilibrium}

In the previous subsection, we have assumed that the $\tilde{\nu}_{R}$ production from the decay of MSSM superparticles in chemical equilibrium is negligible. Indeed, this is the case in most of the parameter region. In some case, however, such a production mechanism also gives sizable amount of right-handed sneutrino. (We denote its contribution to the relic density by $\Omega_{\tilde{\nu}_{R}}^{\mathrm{CE}}$.) In this subsection, we consider $\Omega_{\widetilde{\nu}_{R}}^{\mathrm{CE}}$.

$\Omega_{\tilde{\nu}_{R}}^{\mathrm{CE}}$ is estimated by solving the Boltzmann equation

$$
\frac{d n_{\tilde{\nu}_{R}}}{d t}+3 H n_{\tilde{\nu}_{R}}=C_{\text {decay }},
$$

where $n_{\tilde{\nu}_{R}}$ is the number density of $\tilde{\nu}_{R}$. Denoting the distribution function of particle $x$ in the chemical equilibrium as $f_{x}$, the decay term is given by

$$
C_{\text {decay }}=\sum_{x, y} \int \frac{d^{3} k_{x}}{(2 \pi)^{3}} \gamma_{x}\left(2 s_{x}+1\right) \Gamma_{x \rightarrow \tilde{\nu}_{R} y} f_{x}\left\langle 1 \pm f_{y}\right\rangle_{k_{x}},
$$

where $\gamma_{x}=m_{x} / \sqrt{k_{x}^{2}+m_{x}^{2}}$ is the Lorentz factor, $\left(2 s_{x}+1\right)$ is the spin multiplicity of $x$, and $\left\langle 1 \pm f_{y}\right\rangle_{k_{x}}$ is the averaged final-state multiplicity factor for fixed value of initialstate momentum (with the positive and negative signs being for bosons and fermions, respectively.) In addition, the summation is over all the possible production processes of right-handed sneutrino. Then, the relic density is found as

$$
\Omega_{\tilde{\nu}_{R}}^{\mathrm{CE}}=\frac{m_{\tilde{\nu}_{R}} n_{\tilde{\nu}_{R}, 0}}{\rho_{\mathrm{cr}}},
$$

where $n_{\tilde{\nu}_{R}, 0}$ is the present value of $n_{\tilde{\nu}_{R}}$.

Solution to the above Boltzmann equation has been already studied in Ref. [3]; in Ref. 3], we found that $\Omega_{\tilde{\nu}_{R}}^{\mathrm{CE}}$ becomes much smaller than $\Omega_{\mathrm{DM}}$ with neutrino mass matrix with normal hierarchy if tri-linear scalar coupling is small. Indeed, if $A_{\nu}=0$, the relevant processes of the $\tilde{\nu}_{R}$ production are the decay of Higgsinos, $\tilde{H}^{0} \rightarrow \tilde{\nu}_{R} \bar{\nu}_{L}$ and $\tilde{H}^{+} \rightarrow \tilde{\nu}_{R} l_{L}^{+}$. Then, we found

$$
\Omega_{\tilde{\nu}_{R}}^{\mathrm{CE}} h^{2} \lesssim 1.7 \times 10^{-3}\left(\frac{m_{\nu}^{2}}{2.8 \times 10^{-3} \mathrm{eV}^{2}}\right)
$$

(Here, we assume that the neutral and charged Higgsinos are mass eigenstates with mass $\left|\mu_{H}\right|$.) The above expression is independent of $m_{\tilde{\nu}_{R}}$, and the maximal abundance is obtained when $\left|\mu_{H}\right| \simeq 2.75 m_{\tilde{\nu}_{R}}$.

It is notable that $\Omega_{\tilde{\nu}_{R}}^{\mathrm{EE}}$ can be as large as $\Omega_{\mathrm{DM}}$ when the mass of left-handed sneutrino is close to right-handed sneutrino mass. This is because the left-right mixing angle of sneutrinos is enhanced in such a case. In the case of minimal supergravity model, such a mass spectrum is hardly realized. In general, however, left-handed and right-handed sneutrino masses are free parameters and hence those masses may be degenerate. Thus, in this subsection, we work in the general framework of the MSSM rather than adopting minimal supergravity model. When the mass difference between left-handed and right-handed sneutrinos are small, the right-handed sneutrino production is dominated by the decay of Wino and Bino in the chemical equilibrium: $\tilde{W}^{0} \rightarrow \tilde{\nu}_{R} \bar{\nu}_{L}$, $\tilde{W}^{+} \rightarrow \tilde{\nu}_{R} l_{L}^{+}$, and $\tilde{B} \rightarrow \tilde{\nu}_{R} \bar{\nu}_{L}$ (and their CP conjugated processes). In the following, let us discuss this case approximating that the mass eigenstates of charginos and neutralinos are Wino and Bino (and Higgsinos).

The left-right mixing angle of sneutrinos in the vacuum is given in Eq. (11). Importantly, at high temperature, the mixing angle varies since the expectation value of the Higgs boson depends on the temperature; we estimate the temperature-dependent mixing angle as

$$
\Theta(T)=\frac{1}{2} \tan ^{-1}\left(\frac{2 y_{\nu} v(T)\left|\cot \beta \mu_{H}-A_{\nu}^{*}\right|}{m_{\tilde{\nu}_{L}}^{2}(T)-m_{\tilde{\nu}_{R}}^{2}}\right),
$$

where $v(T)$ is the temperature-dependent expectation value of standard-model-like Higgs boson $H_{\mathrm{SM}}$. Here, we assume that the heavier Higgses have masses much larger than $m_{Z}$ and that the temperature dependence of Higgs mixing angle $\beta$ is negligible. In addition, $m_{\tilde{\nu}_{L}}^{2}(T)$ is the temperature-dependent mass of left-handed sneutrino. With $\Theta(T)$, decay rates of the relevant processes 
are given by

$$
\begin{aligned}
\Gamma_{\tilde{W}^{0} \rightarrow \tilde{\nu}_{R} \bar{\nu}_{L}} & =\frac{g_{2}^{2}}{64 \pi} \Theta^{2}(T) m_{\tilde{W}}\left(1-\frac{m_{\tilde{\nu}_{R}}^{2}}{m_{\tilde{W}}^{2}}\right)^{2}, \\
\Gamma_{\tilde{W}^{+} \rightarrow \tilde{\nu}_{R} l_{L}^{+}} & =\frac{g_{2}^{2}}{32 \pi} \Theta^{2}(T) m_{\tilde{W}}\left(1-\frac{m_{\tilde{\nu}_{R}}^{2}}{m_{\tilde{W}}^{2}}\right)^{2}, \\
\Gamma_{\tilde{B}_{\tilde{\nu}_{R} \bar{\nu}_{L}}} & =\frac{g_{1}^{2}}{64 \pi} \Theta^{2}(T) m_{\tilde{B}}\left(1-\frac{m_{\tilde{\nu}_{R}}^{2}}{m_{\tilde{B}}^{2}}\right)^{2} .
\end{aligned}
$$

In our analysis, we approximate the Higgs potential in thermal bath as 22]

$$
\begin{aligned}
V_{T} \simeq & \frac{m_{h}^{2}}{4 v^{2}}\left(\left|H_{\mathrm{SM}}\right|^{2}-v^{2}\right)^{2} \\
& +\frac{1}{8 v^{2}}\left(2 m_{W}^{2}+m_{Z}^{2}+2 m_{t}^{2}\right) T^{2}\left|H_{\mathrm{SM}}\right|^{2} .
\end{aligned}
$$

Minimizing this potential, we obtain $v(T)$; denoting critical temperature (which is defined as the temperature where the curvature of $V_{T}$ at $H_{\mathrm{SM}}=0$ vanishes) as $T_{C}$, we obtain the expectation value at $T<T_{C}$ as

$$
v(T)=v \sqrt{1-T^{2} / T_{C}^{2}} .
$$

In our calculation, we use $m_{h}=115 \mathrm{GeV}$ and $m_{t}=175$ $\mathrm{GeV}$. In this case, $T_{C}=139 \mathrm{GeV}$.

With the shift of the expectation value of Higgs boson at high temperature, the mixing angle is modified, as shown in Eq. (34). The effects are from the change of the left-right mixing mass of sneutrino, and also from the shift of $m_{\tilde{\nu}_{L}}^{2}(T)$ via the temperature dependence of $D$-term condensation. (The second effect was not considered in Ref. [3].) In this paper, we have included both of these effects and calculated the density parameter of $\tilde{\nu}_{R}$.

In calculating the temperature-dependent mass of lefthanded sneutrino $m_{\tilde{\nu}_{L}}^{2}(T)$, one may think that it is also necessary to take account of the thermal mass of the sneutrino. At the temperature $T>T_{C}$, the thermal mass is from the gauge-boson-loop diagrams, and is estimated as

$$
\delta m_{\tilde{\nu}_{L}}^{2}=\frac{1}{16}\left(3 g_{2}^{2}+g_{1}^{2}\right) T^{2} .
$$

Importantly, however, we are interested in the case where $v(T)$ is non-vanishing since the production of $\tilde{\nu}_{R}$ is effective in such a case. Then, the thermal mass of left-handed sneutrino should be suppressed since gauge bosons acquire masses from the expectation value of the Higgs boson. Detailed calculation of the thermal mass is more complicated in this case and we do not go into the detail. Instead, we perform our analysis with Eq. (40) and with $\delta m_{\tilde{\nu}_{L}}^{2}=0$ (i.e., with neglecting the thermal mass of left-handed sneutrino). As we will see, production of right-handed sneutrino becomes effective at relatively low temperature and $\Omega_{\tilde{\nu}_{R}}^{\mathrm{CE}}$ does not change much between two procedures.

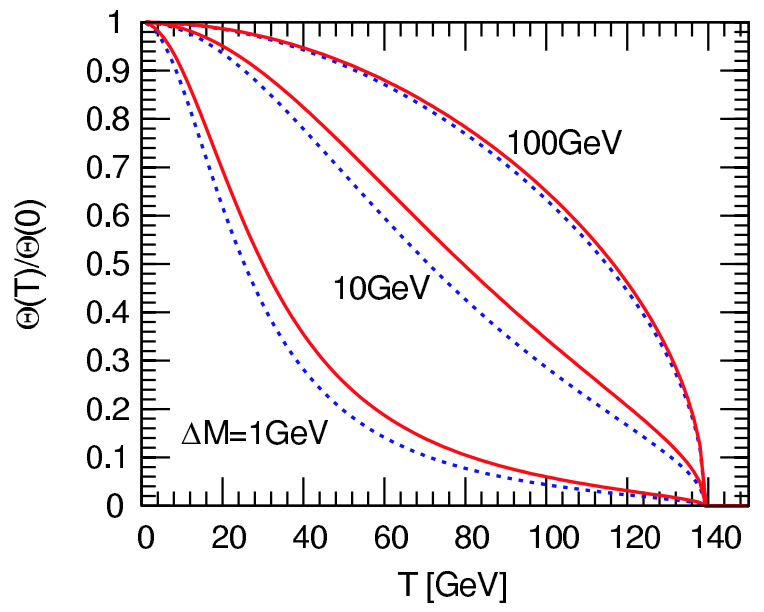

FIG. 7: The left-right mixing angle of sneutrinos at finite temperatures $\Theta(T)$ normalized bu $\Theta(0)$. We take here $m_{\tilde{\nu}_{R}}=$ $100 \mathrm{GeV}$, and the mass difference $\Delta M=m_{\tilde{\nu}_{L}}-m_{\tilde{\nu}_{R}}$ is 1,10 and $100 \mathrm{GeV}$ from left to right. The results obtained by using $\delta m_{\tilde{\nu}_{L}}^{2}=0$ and $\delta m_{\tilde{\nu}_{L}}^{2}$ given in Eq. (40) are shown by the solid and dashed lines, respectively.

In Fig. 7 we show the left-right mixing angle at the finite temperature (relative to the zero-temperature value). We can see that the mixing angle is drastically suppressed when the temperature becomes close to $T_{C}$.

Now, we estimate the relic density of $\tilde{\nu}_{R}$ coming from the decays of superparticle in the chemical equilibrium. We calculate $\Omega_{\tilde{\nu}_{R}}^{\mathrm{CE}}$ by solving Eq. (30). In Fig. 8] we show $\Omega_{\tilde{\nu}_{R}}^{\mathrm{CE}} h^{2}$ as a function of $m_{\tilde{\nu}_{L}}$. As we have mentioned, $\Omega_{\tilde{\nu}_{R}}^{\mathrm{CE}}$ becomes larger as $m_{\tilde{\nu}_{L}}$ gets close to $m_{\tilde{\nu}_{R}}{ }^{7}$ In particular, $\Omega_{\tilde{\nu}_{R}}^{\mathrm{CE}}$ becomes consistent with the density parameter of dark matter with the mass degeneracy of $\sim 10 \%$ for $\left|a_{\nu}\right|<3$. We have also checked that $\Omega_{\tilde{\nu}_{R}}^{\mathrm{CE}}$ decreases as $m_{\tilde{W}}$ increases. This is due to the fact that the sneutrino production at higher temperature becomes ineffective since the left-right mixing angle of sneutrinos is suppressed at high temperature. (See Fig. 9.)

Eq. (33) suggests another possibility of enhancing $\Omega_{\tilde{\nu}_{R}}^{\mathrm{CE}}$. By assuming degenerate neutrino mass, Yukawa coupling constants of neutrinos become larger and hence more $\tilde{\nu}_{R}$ can be produced. With Eq. (33), for example, observed dark-matter density given in Eq. (11) is explained when neutrino masses are larger than $0.39 \mathrm{eV}$ with degenerate neutrino masses. Notice that this value of the neutrino mass is consistent with the upper bound on the neutrino mass derived from the WMAP three-year data.

Up to now, we have discussed the two sources of the $\tilde{\nu}_{R}$ relic density, i.e., $\Omega_{\tilde{\nu}_{R}}^{\mathrm{FO}}$ and $\Omega_{\tilde{\nu}_{R}}^{\mathrm{CE}}$. If both of them are sizable, the total present relic abundance is given by the

\footnotetext{
7 Compared with the previous analysis in Ref. [3], $\Omega_{\tilde{\nu}_{R}}^{\mathrm{CE}} h^{2}$ is reduced since the left-right mixing angle of sneutrinos becomes
} smaller due to the additional thermal effects. 


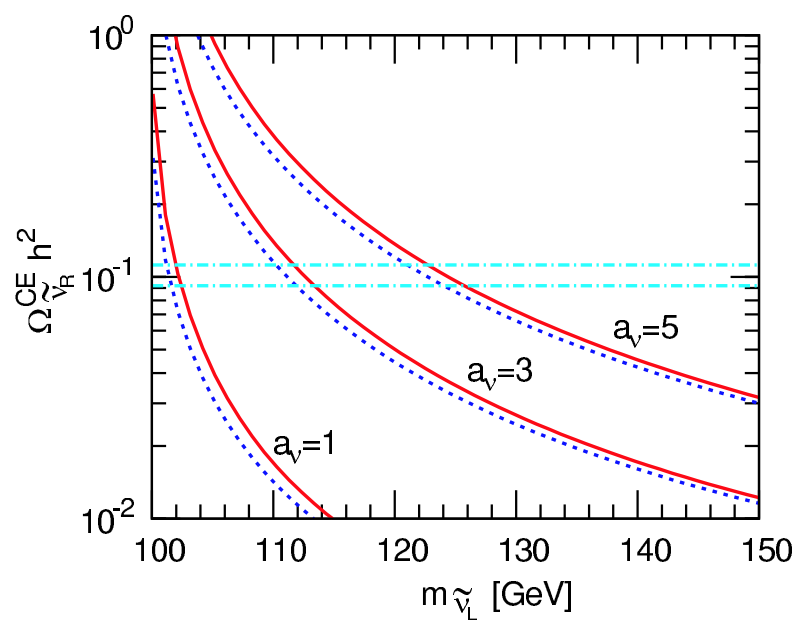

FIG. 8: The relic density $\Omega_{\tilde{\nu}_{R}}^{\mathrm{CE}} h^{2}$ of the LSP $\tilde{\nu}_{R}$ in terms of the left-handed sneutrino mass $m_{\tilde{\nu}_{L}}$. The results obtained by using $\delta m_{\tilde{\nu}_{L}}^{2}=0$ and $\delta m_{\tilde{\nu}_{L}}^{2}$ given in Eq. (40) are shown by the solid and dashed lines, respectively. We take $a_{\nu}=1,3,5$ from left to right. Here $m_{\tilde{\nu}_{R}}=100 \mathrm{GeV}, m_{\tilde{W}}=300 \mathrm{GeV}$ and $\mu_{H}=150 \mathrm{GeV}$. The horizontal dot-dashed lines correspond to the dark-matter density (1).

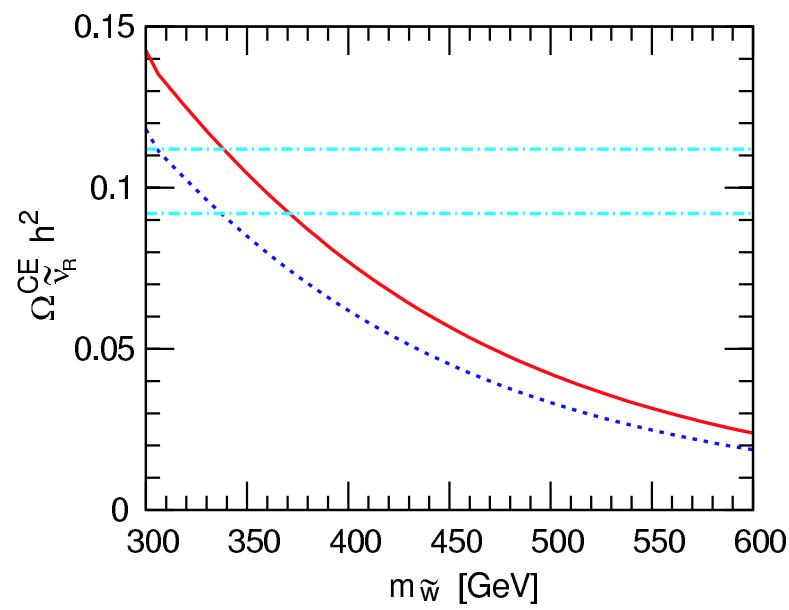

FIG. 9: The relic density $\Omega_{\tilde{\nu}_{R}}^{\mathrm{CE}} h^{2}$ of the LSP $\tilde{\nu}_{R}$ in terms of the wino mass. The results obtained by using $\delta m_{\tilde{\nu}_{L}}^{2}=0$ and $\delta m_{\tilde{\nu}_{L}}^{2}$ given in Eq. (40) are shown by the solid and dashed lines, respectively. We take here $a_{\nu}=3, m_{\tilde{\nu}_{R}}=100 \mathrm{GeV}$, $m_{\tilde{\nu}_{L}}=110 \mathrm{GeV}$ and $\mu_{H}=150 \mathrm{GeV}$. The horizontal dotdashed lines correspond to the dark-matter density (1).

sum of these two contributions:

$$
\Omega_{\tilde{\nu}_{R}}=\Omega_{\tilde{\nu}_{R}}^{\mathrm{FO}}+\Omega_{\tilde{\nu}_{R}}^{\mathrm{CE}}
$$

Before closing this subsection, we comment on the case where the right-handed neutrinos have Majorana mass. If the Majorana mass is smaller than the supersymmetry breaking masses of superparticles, $\tilde{\nu}_{R}$ may still be the LSP. In this case, however, the universe is likely to be overclosed with $\tilde{\nu}_{R}$-LSP. This is because $y_{\nu}$ becomes larger than the value given in Eq. (4) with the Majorana mass. In this case, $\Omega_{\tilde{\nu}_{R}}^{\mathrm{CE}}$ becomes much larger than the value we obtained for the purely Dirac case unless the reheating temperature is so low that superparticles can never be thermalized. (See also [4].)

\section{Other possibilities}

So far, we have discussed the production of $\tilde{\nu}_{R}$ by the decays of MSSM superparticles. In the rest of this section, we briefly comment on other possibilities.

First, $\tilde{\nu}_{R}$ is potentially produced by the decays of nonMSSM superparticles. One interesting candidate is gravitino. Since gravitino has only the gravitationally suppressed interactions, its lifetime is very long and gravitino of mass $\sim 100 \mathrm{GeV}$ decays after BBN epoch. When the primordial abundance of gravitino takes relevant value, its decay produces the correct amount of $\tilde{\nu}_{R}$ and the $\tilde{\nu}_{R^{-}}$ CDM can be realized. In usual cases, decay of the gravitino after BBN is severely constrained by the BBN constraint [20, 23]. However, if the gravitino is the NLSP and also if it decays only into $\tilde{\nu}_{R}+\bar{\nu}\left(\right.$ and $\left.\tilde{\nu}_{R}^{*}+\nu\right)$, the BBN constraints are significantly relaxed. ${ }^{8}$ This is because the branching ratios of gravitino decaying into hadrons and charged particles are very small in this case. We should note that, even in this case, the decay of the MSSM-LSP into gravitino is also constrained from BBN. To avoid it, the MSSM-LSP should dominantly decay into $\tilde{\nu}_{R}$ (and something else) with relatively small hadronic branching ratio or relatively short lifetime.

In addition, in this paper, we have assumed that the initial abundance of $\tilde{\nu}_{R}$ after the reheating of inflation is negligible. Note, however, that we cannot exclude the possibility that $\tilde{\nu}_{R}$ is produced at the reheating (or also at the preheating) epoch by the decay of inflaton. This is possible when $\tilde{\nu}_{R}$ couples to the inflaton although it is highly dependent on the details of the inflation model.

There is yet another possibility. $\tilde{\nu}_{R}$ might be produced as a coherent oscillation. This is because $\tilde{\nu}_{R}$ has an almost flat potential and its initial amplitude after the inflation might be displaced from the origin. In this case, if the initial amplitude is of the order of $10^{9} \mathrm{GeV}$, such coherent mode of $\tilde{\nu}_{R}$ might be the dark-matter assuming that the reheating of the inflation completes before the start of the $\tilde{\nu}_{R}$ oscillation.

\footnotetext{
${ }^{8}$ Similarly, when gravitino is the LSP and $\tilde{\nu}_{R}$ is the NLSP, the gravitino dark matter may be possible without conflicting with the BBN observations.
} 


\section{CONCLUSIONS}

We have considered the scenario where right-handed sneutrino becomes the CDM of the universe in the framework that neutrino masses are purely Dirac-type. We have investigated the production of $\tilde{\nu}_{R}$ by decays of superparticles in the early universe. Especially, the decay of the MSSM-LSP after its freeze-out time have been studied in detail by using the minimal supergravity model of supersymmetry breaking. The NLSP is considered as the MSSM-LSP, $\tilde{\chi}_{1}^{0}$ or $\tilde{\tau}_{1}$.

We have found that there is a wide parameter range in which $\tilde{\nu}_{R}$ is the LSP, and also that the relic density of the LSP $\tilde{\nu}_{R}$ coming from the late MSSM-LSP decay can be consistent with the dark-matter density in such a region. Assuming the minimal supergravity model, the mass of the $\tilde{\nu}_{R}$ is bounded not only from below but also from above. The lower bound comes from the phenomenological constraints (e.g., the Higgs boson mass and the rate of $b \rightarrow s \gamma$ ) or the requirement of $\tilde{\nu}_{R}$ being the LSP. On the other hand, the upper bound comes from the dark-matter density. In addition, in our framework, the MSSM-LSP is the MSSM-LSP is the Bino-like lightest neutralino (almost right-handed lighter stau) when $m_{\tilde{\nu}_{R}}$ is small (large). It should be noted that these features are insensitive to the neutrino mass hierarchy.

We have also shown that the decay of the MSSM-LSP receives constraints from $\mathrm{BBN}$, which leads to the upper bound on the mass of the MSSM-LSP. Indeed, it forbids the MSSM-LSP with mass $\gg 1 \mathrm{TeV}$ in any case. It has been found that, when $\tan \beta=10$ and $\mu_{H}>0$, the $\tilde{\chi}_{1}^{0}$ MSSM-LSP can be consistent with the BBN even with the hierarchical neutrino masses, if the mass of $\tilde{\chi}_{1}^{0}$ is small enough. On the other hand, the $\tilde{\tau}_{1}$ MSSM-LSP is severely constrained from BBN argument. We found that its decay can be consistent with the BBN only when the neutrino masses are degenerate and $m_{\nu}$ is large. How- ever, the BBN bound strongly depends on the lifetime and becomes irrelevant when the lifetime is shorter than about $0.1 \mathrm{sec}$. If we go beyond the assumptions used in our analysis, such a short lifetime can be achieved. In such a case, the decay of the $\tilde{\tau}_{1}$ MSSM-LSP is cosmologically harmless.

When the CDM is indeed $\tilde{\nu}_{R}$, it is not easy to test directly its existence because of very small neutrino Yukawa coupling constants. However, the $\tilde{\nu}_{R}$-CDM scenario has features quite its own, and we will have indirect hints from the future experiments and observations. Since neutrino masses are purely Dirac-type, experiments of the neutrinoless double-beta decay should give null results. The direct searches for dark matter also should give null results. If supersymmetry would be found with the above-mentioned circumstance, $\tilde{\nu}_{R}$ would be regarded as a serious candidate of the LSP and CDM. In addition, the $\tilde{\nu}_{R}$ dark matter scenario may be distinguishable from the conventional scenario where the $\tilde{\chi}_{1}^{0}$ MSSM-LSP is dark matter. One interesting feature of the $\tilde{\nu}_{R}$-CDM is that the MSSM-LSP can be charged (or even colored), e.g., $\tilde{\tau}_{1}$ as we have considered. Even if the MSSM-LSP is $\tilde{\chi}_{1}^{0}$, as we have shown, the mass relations between $m_{\tilde{\chi}_{1}^{0}}$ and $m_{\tilde{\tau}_{1}}$ and also between $m_{\tilde{\chi}_{1}^{+}}$and $m_{\tilde{\tau}_{1}}$ are significantly different in these two dark-matter scenarios. These differences will be testable in the precise measurements of superparticles at future collider experiments.

\section{Acknowledgments}

The work was partially supported by the grants-inaid from the Ministry of Education, Science, Sports, and Culture of Japan, Nos. 16081202, 17340062 and 18740122 (T.A.), and No. 15540247 (T.M.).
[1] D. N. Spergel et al., astro-ph/0603449.

[2] See, for example, G. Jungman, M. Kamionkowski and K. Griest, Phys. Rept. 267, 195 (1996) hep-ph/9506380.

[3] T. Asaka, K. Ishiwata and T. Moroi, Phys. Rev. D 73, 051301 (2006) hep-ph/0512118.

[4] S. Gopalakrishna, A. de Gouvea and W. Porod, JCAP 0605, 005 (2006) hep-ph/0602027.

[5] J. McDonald, hep-ph/0609126.

[6] S. Fukuda et al. [Super-Kamiokande Collaboration], Phys. Lett. B 539, 179 (2002) hep-ex/0205075.

[7] Y. Ashie et al. [Super-Kamiokande Collaboration], Phys. Rev. D 71, 112005 (2005) hep-ex/0501064.

[8] E. Aliu et al. [K2K Collaboration], Phys. Rev. Lett. 94, 081802 (2005) hep-ex/0411038.

[9] S. N. Ahmed et al. [SNO Collaboration], Phys. Rev. Lett. 92, 181301 (2004) nucl-ex/0309004.

[10] T. Araki et al. [KamLAND Collaboration], Phys. Rev. Lett. 94, 081801 (2005) hep-ex/0406035.

[11] T. Yanagida, in "Proceedings of the Workshop on Uni- fied Theory and Baryon Number of the Universe," eds. O. Sawada and A. Sugamoto (KEK, 1979) p.95; M. GellMann, P. Ramond and R. Slansky, in "Supergravity," eds. P. van Niewwenhuizen and D. Freedman (North Holland, 1979); S. L. Glashow, in "Proceedings of the Cargése Summer Institute on Quarks and Leptons," (Plenum, 1980) p707.

[12] G. 't Hooft, in "Recent Developments in Gauge Theories," (Plenum, 1980) p.135.

[13] A. H. Chamseddine, R. Arnowitt and P. Nath, Phys. Rev. Lett. 49, 970 (1982); R. Barbieri, S. Ferrara and C. A. Savoy, Phys. Lett. B 119, 343 (1982); L. J. Hall, J. D. Lykken and S. Weinberg, Phys. Rev. D 27 (1983) 2359.

[14] M. Fukugita, K. Ichikawa, M. Kawasaki and O. Lahav, Phys. Rev. D 74, 027302 (2006) astro-ph/0605362.

[15] T. Moroi and L. Randall, Nucl. Phys. B 570, 455 (2000) arXiv:hep-ph/9906527.

[16] G. Belanger, F. Boudjema, A. Pukhov and A. Se- 
menov, Comput. Phys. Commun. 149, 103 (2002) hep-ph/0112278; Comput. Phys. Commun. 174, 577 (2006) hep-ph/0405253.

[17] W. M. Yao et al. [Particle Data Group], J. Phys. G 33, 1 (2006).

[18] [Heavy Flavor Averaging Group (HFAG) Collaboration], hep-ex/0603003.

[19] T. Asaka, K. Hamaguchi and K. Suzuki, Phys. Lett. B 490, 136 (2000) hep-ph/0005136.

[20] For example, see a recent analysis by M. Kawasaki, K. Kohri and T. Moroi, Phys. Lett. B 625, 7 (2005)
arXiv:astro-ph/0402490; Phys. Rev. D 71, 083502 (2005) astro-ph/0408426.

[21] Y. I. Izotov and T. X. Thuan, Astrophys. J. 602, 200 (2004) astro-ph/0310421.

[22] M. Dine, R. G. Leigh, P. Y. Huet, A. D. Linde and D. A. Linde, Phys. Rev. D 46, 550 (1992) arXiv:hep-ph/9203203.

[23] K. Kohri, T. Moroi and A. Yotsuyanagi, Phys. Rev. D 73, 123511 (2006) arXiv:hep-ph/0507245. 